\title{
Indoleamine 2,3-dioxygenase in endometrial cancer: a targetable mechanism of immune resistance in mismatch repair-deficient and intact endometrial carcinomas
}

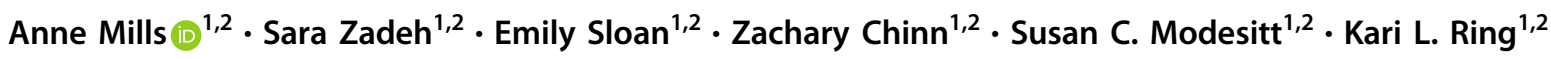

Received: 11 October 2017 / Revised: 22 January 2018 / Accepted: 25 January 2018 / Published online: 20 March 2018

(c) United States \& Canadian Academy of Pathology 2018

\begin{abstract}
Mismatch repair-deficient endometrial carcinomas are optimal candidates for immunotherapy given their high neoantigen loads, robust lymphoid infiltrates, and frequent PD-L1 expression. However, co-opting the PD-1/PD-L1 pathway is just one mechanism that tumors can utilize to evade host immunity. Another immune modulatory molecule that has been demonstrated in endometrial carcinoma is indoleamine 2,3-dioxygenase (IDO). We herein evaluate IDO expression in 60 endometrial carcinomas and assess results in relation to PD-L1 and mismatch repair status. IDO immunohistochemistry was performed on 60 endometrial carcinomas (20 Lynch syndrome (LS)-associated, 20 MLH1 promoter hypermethylated, and 20 mismatch repair-intact). Eight-five percent of endometrial carcinomas showed IDO tumor staining in $>1 \%$ of cells. Twenty-five percent were positive in $>25 \%$ of tumor cells and only $7 \%$ exceeded $50 \%$ staining. Mismatch repair-deficient cancers were more likely than mismatch repair-intact cancers to be $>25 \%$ IDO-positive ( $35 \%$ vs. $5 \% p=0.024)$. Differences were amplified when Lynch syndrome-associated cases were evaluated in isolation (50\% Lynch syndrome-associated vs. $10 \%$ mismatch repair-intact and MLH1-hypermethylated, $p=0.001$ ). Of the four cases showing $>50 \%$ staining, three were Lynch syndrome-associated and one was MLH1-hypermethylated; no mismatch repair-intact cases had $>50 \%$ staining. Forty-three percent of IDO-positive tumors were also positive for PD-L1, whereas only two cases showed tumoral PD-L1 in the absence of IDO. In summary, IDO expression is prevalent in endometrial carcinomas and diffuse staining is significantly more common in mismatch repairdeficient cancers, particularly Lynch syndrome-associated cases. Given that the majority of PD-L1 positive cancers also express IDO, synergistic combination therapy with anti-IDO and anti-PD1/PD-L1 may be relevant in this tumor type. Furthermore, anti-IDO therapy may be an option for a small subset of mismatch repair-intact cancers.
\end{abstract}

\section{Introduction}

Mismatch repair-deficient endometrial carcinomas are ideal candidates for immunotherapy given their prominent tumorassociated cytotoxic $\mathrm{T}$ cells, elevated neoantigen loads, and propensity to express PD-L1 [1-11]. Checkpoint inhibitors targeting the PD-1/PD-L1 axis have shown promise in this tumor type, with robust responses in initial clinical trials [2].

Anne Mills

amm7r@virginia.edu

1 Department of Pathology, University of Virginia, Charlottesville VA USA

2 Department of Gynecology and Obstetrics, Division of Gynecologic Oncology, University of Virginia, Charlottesville VA USA
However, co-opting the PD-L1 pathway is only one of many defense mechanisms that tumors can mount to evade host immunity, and interference with this checkpoint may not be independently curative in cancers with multiple modalities of immune resistance [11-13]. Another immune modulatory molecule that has been demonstrated in endometrial carcinomas is indoleamine 2,3-dioxygenase (IDO) [14].

IDO is an enzyme that interferes with T-cell function in two ways: first, it depletes tryptophan, which is necessary for T-cell survival, and second, it generates the toxic metabolite kynurenine [15]. IDO was first discovered on placental trophoblasts where it promotes maternal-fetal tolerance. In its normal function, it can also play an immunosuppressive role following inflammatory stimuli to prevent perpetual immune activation and attendant immunopathology [15]. However, like the checkpoint molecule PD-L1, IDO can also be pirated by malignancies as a mechanism of immune escape. 
IDO has been identified in endometrial carcinomas where it has been shown to interfere with lymphocytic tumor infiltration and correlate with a worsened prognosis [16-18]. Recently developed IDO inhibitors have shown some early promise in cancer therapy and include 1-methyl-DL-tryptophan, small molecule IDO inhibitors, and IDO peptide vaccines [15]. Such approaches may have utility either as monotherapies or as adjuvants to other immunotherapies, including anti-PD-1/PD-L1 checkpoint inhibitors. However, the potential efficacy of such approaches remains unknown in the endometrium as the relationship between IDO and PDL1 expression in endometrial carcinomas has not been studied, nor has IDO expression specifically been assessed with attention to mismatch repair status at this site. We herein evaluated IDO expression with relation to PD-L1 and mismatch repair status in a cohort of 60 endometrial carcinomas including Lynch syndrome-associated, MLH1 promoter hypermethylated, and mismatch repair-intact cases.

\section{Methods}

\section{Case selection}

This retrospective study was approved by the institutional review board of the University of Virginia. Whole sections of formalin-fixed, paraffin embedded tissue from 20 Lynch syndrome-associated carcinomas, 20 MLH1 promoter hypermethylated carcinomas, and 20 mismatch repair-intact carcinomas were assembled for evaluation. Clinical characteristics were abstracted from the electronic medical record.

\section{Mismatch repair status}

Lynch syndrome-associated and MLH1 promoter hypermethylated cases were classified as such at the time of original diagnosis through the universal Lynch syndrome screening program using immunohistochemistry for the four mismatch repair proteins MLH1 (clone ES05, predilute; Leica Biosystems), PMS2 (clone MRG-28Mab, predilute; Cell Marque), MSH2 (clone 25D12, predilute; Leica), and MSH6 (clone 44 Mab, predilute; Cell Marque). Lynch syndrome-associated cases were defined as cases with total nuclear loss of expression for the mismatch repair proteins MSH2, MSH6, and/or PMS2 with confirmatory germline testing results when performed. All $M L H 1$ promoter hypermethylated cases showed complete dual loss of nuclear staining for MLH1/PMS2 by immunohistochemistry with methylation confirmation on PCR.

\section{IDO and PD-L1 immunohistochemistry}

Immunohistochemical staining for IDO (Sigma Prestige, HPA 023072, 1:2000 dilution) and PD-L1/CD274 (Spring
Biosciences, SP142, 1:200 dilution; internally validated against Dako 22C3 clone) was performed on all cases; PDL1 results for the majority of the cases (17 Lynch syndrome-associated, 18 MLH1-hypermethylated, and 19 mismatch repair-intact cases) had previously been reported in a prior publication; other cases from that series were not included because whole section unstained slides were not available for further investigations [4]. Both immunohistochemical stains were scored in both the tumor and the peritumoral immune compartment (e.g., the "immune stroma"). Staining was scored manually at the microscope by two pathologists (A.M.M. and S.Z.) using dual-headed microscope review and consensus agreement on percentage positivity. Tumor staining was classified as positive when clear membranous (PD-L1) or cytoplasmic (IDO) staining was present in $\geq 1 \%$ of tumor cells. Tumor staining was classified as positive when clear membranous staining was present in $\geq 1 \%$ of tumor cells. Staining extent was further characterized in the following subcategories: 1-5, $6-10,11-25,26-50$, and $>50 \%$. The $1 \%$ threshold for positivity was selected based on data demonstrating clinical response to PD-L1 inhibition at this expression level in some cancers, and percentage categories were further selected to include the cutoffs for all available PD-L1 inhibitors [5, 19]. Because there are no clinically accepted thresholds for IDO expression, the same cut-points were used for this stain. Immune microenvironment (e.g., "immune stromal") staining was scored as positive when $\geq 5 \%$ of peritumoral and intratumoral immune cells (including lymphocytes and macrophages) showed reactivity and was further subdivided by extent as 5-10, 11-25, 26-50, and $>50 \%$. The 5\% lower limit was selected because single, scattered PD-L1 and IDO-positive inflammatory cells were observed in benign control tissues.

\section{Statistics}

Descriptive statistics were calculated for variables of interest. Statistical analysis was performed using the two-tiered Fischer exact test for categorical variables and a one-way analysis of variance for continuous variables with SPSS statistics, version 24 (IBM, Armonk, NY).

\section{Results}

\section{Case distribution}

There were no significant differences in the distribution of clinical characteristics across the mismatch repair categories (Table 1). Lynch syndrome-associated cancers presented at a younger age (58 years vs. 68 years for MLH1- 
Table 1 Case distribution

\begin{tabular}{|c|c|c|c|c|}
\hline Clinical characteristics & $\begin{array}{l}\text { MMR-deficient LS- } \\
\text { associated } N=20 \\
(\%)\end{array}$ & $\begin{array}{l}\text { MMR-deficient } M L H 1 \text { - } \\
\text { hypermethylated } N=20(\%)\end{array}$ & $\begin{array}{l}\text { MMR-intact } \\
N=20(\%)\end{array}$ & $p$ value \\
\hline $\begin{array}{l}\text { Age at diagnosis, mean } \\
\text { (SD) }\end{array}$ & $58.4(11.1)$ & $67.9(8.6)$ & $65.2(10.8)$ & $p=0.63$ \\
\hline Stage & & & & $p=0.52$ \\
\hline IA & $15(75)$ & $10(50)$ & $15(75)$ & \\
\hline IB & $2(10)$ & $6(30)$ & $3(15)$ & \\
\hline II & $0(0)$ & $1(5)$ & $0(0)$ & \\
\hline IIIA & $1(5)$ & $0(0)$ & $0(0)$ & \\
\hline IIIB & $0(0)$ & $1(5)$ & $0(0)$ & \\
\hline IIIC1 & $1(5)$ & $1(5)$ & $2(10)$ & \\
\hline IIIC2 & $1(5)$ & $1(5)$ & $0(0)$ & \\
\hline Grade & & & & $p=0.06$ \\
\hline 1 & $11(55)$ & $4(20)$ & $9(45)$ & \\
\hline 2 & $2(10)$ & $10(50)$ & $7(35)$ & \\
\hline $3 *$ & $7(35)$ & $6(30)$ & $4(20)$ & \\
\hline Adjuvant treatment & & & & $p=0.60$ \\
\hline Surveillance & $12(60)$ & $9(45)$ & $8(40)$ & \\
\hline Vaginal cuff brachytherapy & $6(30)$ & $7(35)$ & $8(40)$ & \\
\hline $\begin{array}{l}\text { Vaginal cuff brachytherapy } \\
\text { and chemotherapy }\end{array}$ & $1(5)$ & $0(0)$ & $0(0)$ & \\
\hline Chemotherapy & $0(0)$ & $1(5)$ & $1(5)$ & \\
\hline $\begin{array}{l}\text { Chemotherapy and pelvic } \\
\text { radiation }\end{array}$ & $1(5)$ & $2(10)$ & $0(0)$ & \\
\hline Pelvic radiation & $0(0)$ & $1(5)$ & $2(10)$ & \\
\hline Hormones & $0(0)$ & $0(0)$ & $1(5)$ & \\
\hline Recurrence & & & & $p=0.43$ \\
\hline Yes & $1(5)$ & $1(5)$ & $3(15)$ & \\
\hline No & $18(90)$ & $18(90)$ & $14(50)$ & \\
\hline Unknown & $1(5)$ & $1(5)$ & $3(15)$ & \\
\hline Vital status & & & & $p=0.18$ \\
\hline Alive, NED & $17(85)$ & $20(100)$ & $16(80)$ & \\
\hline Alive with disease & $1(5)$ & $0(0)$ & $0(0)$ & \\
\hline Alive, unknown disease & $1(5)$ & $0(0)$ & $0(0)$ & \\
\hline Dead with disease & $0(0)$ & $0(0)$ & $3(15)$ & \\
\hline Dead, unknown disease & $1(5)$ & $0(0)$ & $1(5)$ & \\
\hline
\end{tabular}

* Includes both grade 3 endometrioid and de-differentiated tumors

hypermethylated and 65 years for mismatch repair-intact) (Fig. 1).

\section{IDO expression}

Tumoral IDO expression was common in endometrial carcinomas (Fig. 2), with $85 \%$ (51/60) of all cases showing tumor staining above the $1 \%$ threshold and $60 \%(36 / 60)$ showing staining above the $5 \%$ threshold (Table 2). Most tumors showed positivity concentrated at the infiltrating edge. $25 \%(15 / 60)$ of cases were positive in $>25 \%$ of cells and only $7 \%$ (4/60) exceeded the 50\% staining threshold (Fig. 3). While there were no significant differences in IDO expression by mismatch repair status using the $1 \%$ threshold, at the $25 \%$ threshold, mismatch repair-deficient (either Lynch syndrome-associated or methylated) cancers were more likely than mismatch repair-intact cancers to be IDOpositive $(35 \%(14 / 40)$ vs. $5 \%(1 / 20), p=0.024)$. The mismatch repair-based differences in IDO expression at the $25 \%$ threshold were accentuated when Lynch syndromeassociated cases were evaluated in isolation $(50 \%(10 / 10)$ Lynch syndrome-associated vs. $10 \%$ (4/40) mismatch 

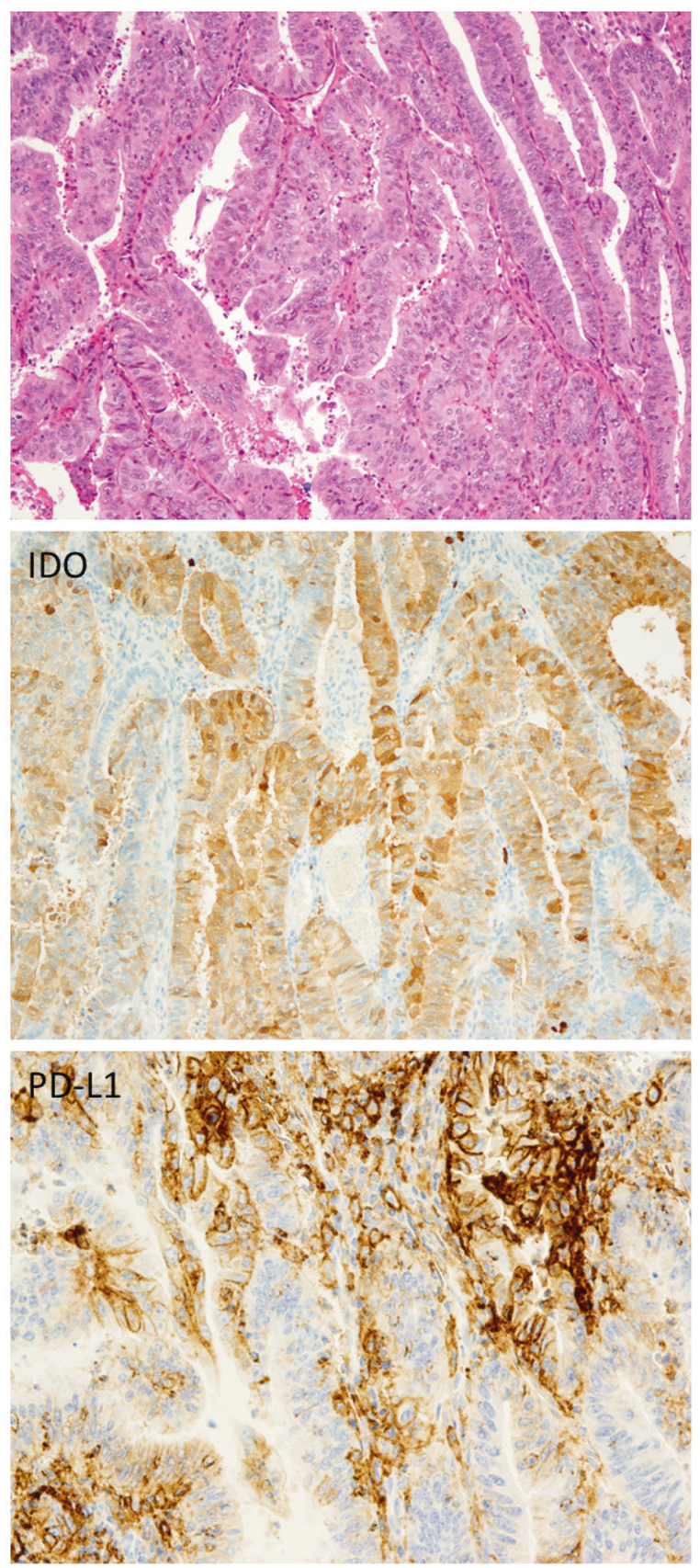

Fig. 1 A Lynch syndrome-associated mismatch repair-deficient endometrial carcinoma with diffuse $(>50 \%)$ tumoral expression of IDO with areas of strong PD-L1 co-expression (10\% overall). Scattered tumor-associated lymphocytes and macrophages are also positive for both markers. This tumor derived from a patient with a germline MSH2 mutation (case 1 in Table 4)

repair-intact and MLH1-hypermethylated, $p=0.001$ ). Of the four cases showing $>50 \%$ staining, three were Lynch syndrome-associated and one was MLH1-hypermethylated; no mismatch repair-intact cases had $>50 \%$ staining (Table 2).

Immune IDO expression was also present in the majority of cases $(82 \%, 49 / 60)$ across mismatch repair subsets,
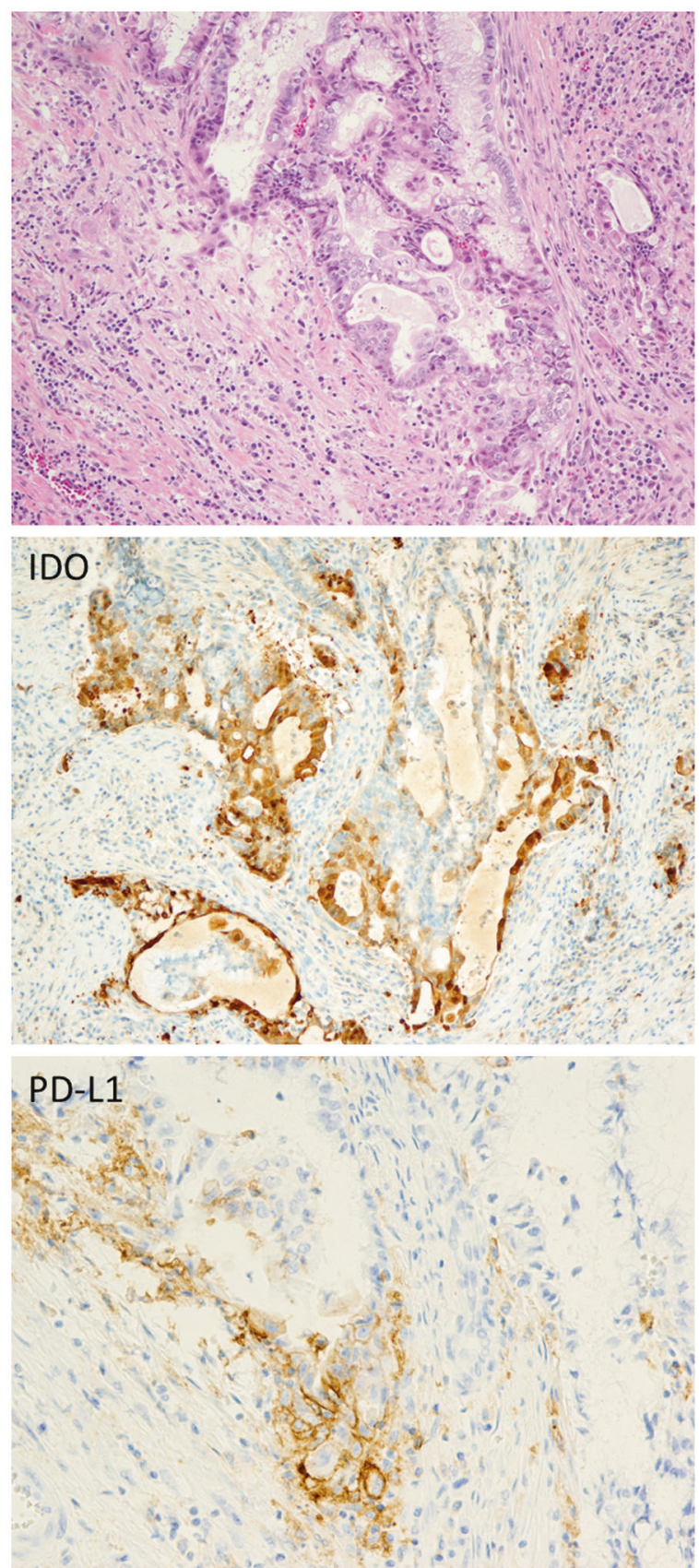

Fig. 2 An $M L H 1$-hypermethylated mismatch repair-deficient endometrial carcinoma with IDO and PD-L1 expression concentrated at the infiltrating tumor edge. Overall, this tumor showed 25\% tumoral positivity for IDO and $10 \%$ tumoral positivity for PD-L1; occasional immune cells also showed positivity for both markers in regions of infiltration

without significant differences between the three mismatch repair categories using any of the thresholds for staining (Table 2). Diffuse immune cell staining was uncommon, with only one case in the series showing IDO positivity in $>25 \%$ of tumor cells (a stage 1A de-differentiated endometrial carcinoma with PMS2 loss and confirmed germline mutation). 
Table 2 IDO and PD-L1 expression by MMR status

\begin{tabular}{|c|c|c|c|c|}
\hline Expression & $\begin{array}{l}\text { MMR- } \\
\text { deficient } \\
\text { Lynch } \\
\text { syndrome- } \\
\text { associated } N \\
=20(\%)\end{array}$ & $\begin{array}{l}\text { MMR-deficient } \\
M L H 1 \text { - } \\
\text { hypermethylated } N \\
=20(\%)\end{array}$ & $\begin{array}{l}\text { MMR- } \\
\text { intact } N \\
=20 \\
(\%)\end{array}$ & $\begin{array}{l}p \\
\text { value }\end{array}$ \\
\hline $\begin{array}{l}\text { Tumoral } \\
\text { IDO }\end{array}$ & & & & $\begin{array}{l}p= \\
0.09\end{array}$ \\
\hline 0 & $3(15)$ & $1(5)$ & $5(25)$ & \\
\hline 1 & $3(15)$ & $6(30)$ & $6(30)$ & \\
\hline 2 & $2(10)$ & $2(10)$ & $4(20)$ & \\
\hline 3 & $2(10)$ & 7 (35) & $4(20)$ & \\
\hline 4 & 7 (35) & $3(15)$ & $1(5)$ & \\
\hline 5 & $3(15)$ & $1(5)$ & $0(0)$ & \\
\hline $\begin{array}{l}\text { Immune } \\
\text { IDO }\end{array}$ & & & & $\begin{array}{l}p= \\
0.19\end{array}$ \\
\hline 0 & $2(10)$ & $4(20)$ & $5(25)$ & \\
\hline 1 & $6(30)$ & $13(65)$ & $8(40)$ & \\
\hline 2 & $9(45)$ & $3(15)$ & $6(30)$ & \\
\hline 3 & $2(10)$ & $0(0)$ & $1(5)$ & \\
\hline 4 & $1(5)$ & $0(0)$ & $0(0)$ & \\
\hline 5 & $0(0)$ & $0(0)$ & $0(0)$ & \\
\hline $\begin{array}{l}\text { Tumoral } \\
\text { PD-L1 }\end{array}$ & & & & $\begin{array}{l}p= \\
0.006\end{array}$ \\
\hline 0 & $5(25)$ & $12(60)$ & $19(95)$ & \\
\hline 1 & $7(35)$ & $3(15)$ & $1(5)$ & \\
\hline 2 & $6(30)$ & $3(15)$ & $0(0)$ & \\
\hline 3 & $1(5)$ & $1(5)$ & $0(0)$ & \\
\hline 4 & $1(5)$ & $1(5)$ & $0(0)$ & \\
\hline 5 & $0(0)$ & $0(0)$ & $0(0)$ & \\
\hline $\begin{array}{l}\text { Immune } \\
P D-L 1\end{array}$ & & & & $\begin{array}{l}p= \\
0.015\end{array}$ \\
\hline 0 & $0(0)$ & $0(0)$ & $6(30)$ & \\
\hline 1 & $6(30)$ & $6(30)$ & $5(25)$ & \\
\hline 2 & $10(50)$ & 7 (35) & $7(35)$ & \\
\hline 3 & $4(20)$ & $7(35)$ & $2(10)$ & \\
\hline 4 & $0(0)$ & $0(0)$ & $0(0)$ & \\
\hline 5 & $0(0)$ & $0(0)$ & $0(0)$ & \\
\hline
\end{tabular}

$0=<1 \%, \quad 1=1-5 \%, 2=6-10 \%, 3=11-25 \%, 4=26-50 \%, 5=$ $>50 \%$

\section{PD-L1 expression}

PD-L1 data for some of this cohort was previously reported in a prior study [4], however this series includes several additional cases and data (and excludes several cases from the prior study for which additional whole sections were not available). Forty percent (24/60) of all tumors were PD-L1positive in at least $1 \%$ of cells. Positivity was most often seen at the infiltrating edge of the tumor. As was demonstrated in the prior study, PD-L1 tumor staining above the $1 \%$ threshold was most common in Lynch syndrome-

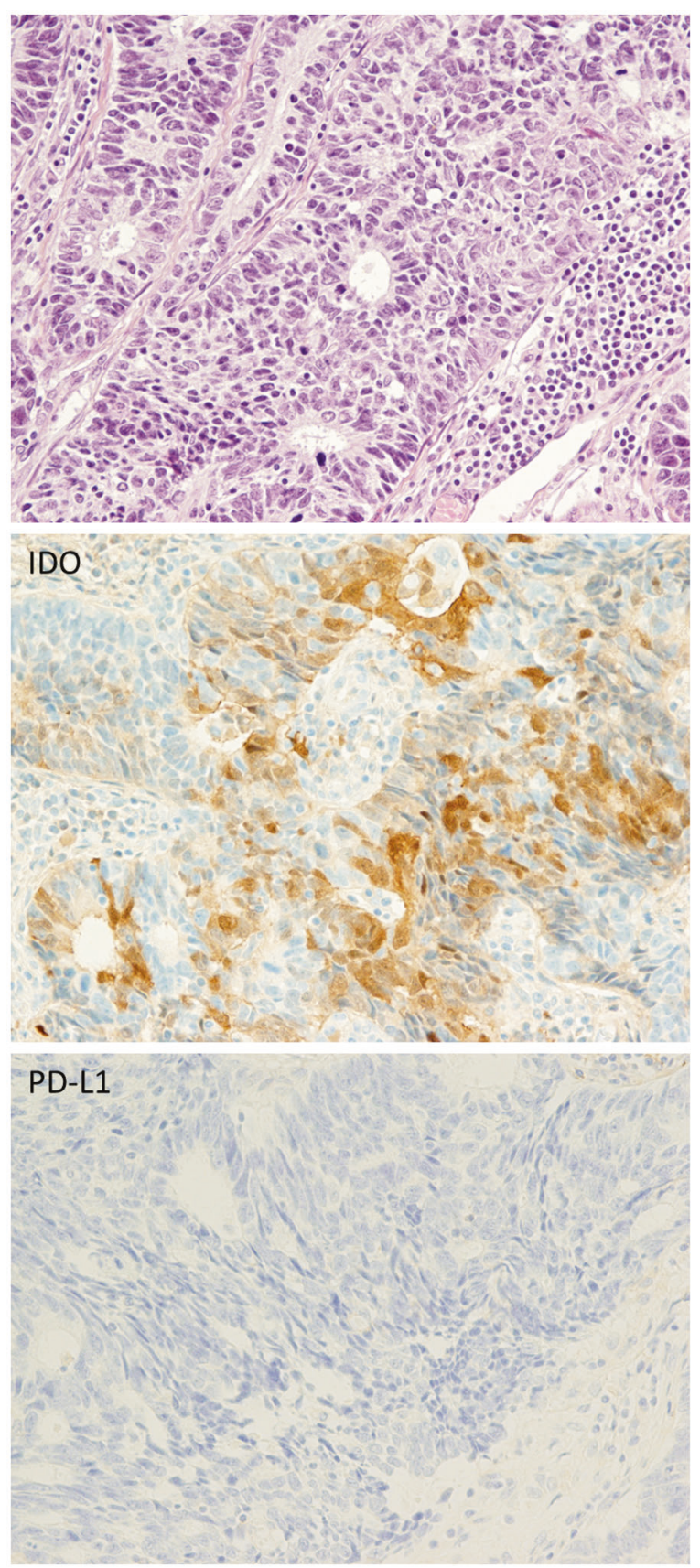

Fig. 3 A mismatch repair-intact endometrial carcinoma with regions of strong tumoral IDO expression (comprising $10 \%$ of overall tumor volume) and complete negativity for PD-L1

associated cancers $(75 \%, 15 / 20)$ followed by MLH1hypermethylated $(40 \%, 8 / 20)$ cancers, and, lastly, mismatch repair-intact cancers $(5 \%, 1 / 20)$. The increased incidence of tumor PD-L1 positivity in mismatch repairdeficient vs. intact carcinomas was statistically significant $((58 \%(23 / 40)$ vs. $5 \%(1 / 20), p=<0.001))$, as was the difference between Lynch syndrome-associated and MLH1hypermethylated cancers $(75 \%(15 / 20)$ vs. $40 \%(8 / 20), p=$ 0.01 ). Only two cases in the series (one Lynch syndrome- 
associated case with MSH6 loss and a confirmed germ line mutation and one MLH1-hypermethylated case) showed $>25 \%$ PD-L1 staining in tumor cells and no cases were positive in $>50 \%$ of tumor cells (Table 2 ).

Immune cell staining for PD-L1 was present in 90\% (54/ 60) of cases: $100 \%$ (40/40) mismatch repair-deficient tumors and $70 \%(14 / 20)$ of intact tumors. The increased incidence of immune cell staining for PD-L1 in mismatch repair-deficient vs. intact cancers was statistically significant $(p=<0.001)$. Greater than $>25 \%$ immune cell staining was not identified in any case.

\section{Relationship between IDO expression and clinicopathologic characteristics}

There was no difference in tumoral or immune cell IDO expression across tumor grade or stage (Tables $3 \mathrm{~A}$ and $3 \mathrm{~B}$ ), however the majority of cases presented with early stage disease $(n=51,85 \%)$. Similarly, there was no difference in recurrence or survival based on IDO status, however only 5 patients (8\%) experienced a recurrence of their disease and 53 patients $(88 \%)$ were alive without disease at last followup.

\section{Relationship between IDO and PD-L1 expression}

Tumoral IDO expression was more common than tumoral PD-L1 expression (85\% (51/60) vs. 40\% (24/60)). Immune cell staining was fairly comparable for both markers $(82 \%$ (49/60) IDO vs. 90\% (54/60) PD-L1). Overall differences in tumoral and immune positivity for IDO and PD-L1 were not statistically significant.

Forty-three percent (22/51) of IDO-positive tumors were also positive for PD-L1, whereas only two cases demonstrated tumoral PD-L1 in the absence of IDO. Dual positivity in tumor cells was identified in a subset of all mismatch repair-deficient cancers and was more common in Lynch syndrome-associated vs. MLH1-hypermethylated tumors, however this was not statistically significant $(70 \%$ $(14 / 20)$ vs. $40 \%(8 / 20), p=0.1))$. Among mismatch repairdeficient cases, 13 IDO-positive tumors were PD-L1-

Table 3A IDO and PD-L1 status by tumor grade

\begin{tabular}{lllll}
\hline Expression & $\begin{array}{l}\text { Grade } 1 N \\
=24(\%)\end{array}$ & $\begin{array}{l}\text { Grade } 2 N \\
=18(\%)\end{array}$ & $\begin{array}{l}\text { Grade } 3 * N \\
=18(\%)\end{array}$ & $p$ value \\
\hline Tumoral IDO & $22(92)$ & $15(83)$ & $17(94)$ & $p=0.16$ \\
Immune IDO & $19(80)$ & $13(72)$ & $17(94)$ & $p=0.26$ \\
Tumoral PD- & $9(38)$ & $6(33)$ & $12(67)$ & $p=0.78$ \\
L1 & & $16(89)$ & $17(94)$ & $p=0.78$ \\
Immune PD- & $21(88)$ & & & \\
L1 & & &
\end{tabular}

* Includes both grade 3 endometrioid and de-differentiated tumors
Table 3B IDO and PD-L1 status by stage

\begin{tabular}{lllll}
\hline Expression & $\begin{array}{l}\text { Stage I } N= \\
51(\%)\end{array}$ & $\begin{array}{l}\text { Stage II } N \\
=1(\%)\end{array}$ & $\begin{array}{l}\text { Stage III } N \\
=8(\%)\end{array}$ & $p$ value \\
\hline Tumoral IDO & $44(86)$ & $1(100)$ & $6(75)$ & $p=0.65$ \\
Immune IDO & $42(82)$ & $1(100)$ & $6(75)$ & $p=0.79$ \\
Tumoral PD- & $20(39)$ & $0(0)$ & $4(50)$ & $p=0.60$ \\
L1 & & $1(100)$ & $7(88)$ & $p=0.92$ \\
Immune PD- & $46(90)$ & & & \\
L1 & & & & \\
\hline
\end{tabular}

negative (3 Lynch syndrome-associated and 10 MLH1methylated), while 1 IDO-negative tumor was PD-L1positve. All four cases that showed diffuse tumoral IDO staining $(>50 \%)$ were also PD-L1 Positive in either the tumor and/or the immune compartment, however none showed tumoral PD-L1 staining $>10 \%$ (Table 4). No mismatch repair-intact cases showed dual staining within tumor cells; the single PD-L1-positive mismatch repair-intact case was IDO-negative.

Dual staining was more common in immune compartment across all mismatch repair groups, with $90 \%(18 / 20)$ of Lynch syndrome-associated, 80\% (16/20) of MLH1hypermethlayed, and 55\% (11/20) of mismatch repair-intact cases expressing both IDO and PD-L1 within the tumorassociated immune cells; these differences were not statistically significant. Six mismatch repair-deficient cases showed PD-L1-only immune staining (two Lynch syndrome-associated and four MLH1-hypermethylated cases), whereas no mismatch repair-deficient cases were positive only for IDO in the immune compartment. In contrast, there were four mismatch repair-intact cases with IDO-only immune staining and three cases with PD-L1only immune staining.

\section{Discussion}

There has been considerable excitement surrounding the use of immunotherapy to target the PD-1/PD-L1 checkpoint axis in mismatch repair-deficient cancers $[1-3,7,8,13,20]$. This enthusiasm has been stoked by the recent FDAapproval of the PD-1 inhibitor pembrolizumab in solid tumors exhibiting loss of mismatch repair protein expression and/or microsatellite instability [2]. The data for antiPD-1/PD-L1 efficacy in these cancers is promising, however that promise is somewhat tempered by the complexity of the immune milieu in which these drugs intervene. Tumoral expression of PD-L1 is just one of many potential mechanisms of immune evasion, and there are little data on how the concomitant presence of other anti-immune defenses could interfere with effectiveness of anti-PD-1/ PD-L1 therapies in solid cancers, including endometrial 
Table 4 Tumors with $>50 \%$ IDO expression

\begin{tabular}{|c|c|c|c|c|c|c|}
\hline Case & Age at diagnosis & Stage & Grade and histology & IDO expression & PD-L1 expression & MMR status \\
\hline 1 & 44 & IIIC2 & Grade 1 endometrioid & $\begin{array}{l}\text { Tumor: }>50 \% \\
\text { immune: } 6-10 \%\end{array}$ & $\begin{array}{l}\text { Tumor: } 6-10 \% \\
\text { immune: } 11-25 \%\end{array}$ & MSH2/6 loss, confirmed $M S H 2$ mutation \\
\hline 2 & 57 & IIIA & Grade 1 endometrioid & $\begin{array}{l}\text { Tumor: }>50 \% \\
\text { immune: } 1-5 \%\end{array}$ & $\begin{array}{l}\text { Tumor: } 1-5 \% \\
\text { immune: } 6-10 \%\end{array}$ & PMS2 loss, germ line untested \\
\hline 3 & 39 & IA & Grade 2 endometrioid & $\begin{array}{l}\text { Tumor: }>50 \% \\
\text { immune: } 1-5 \%\end{array}$ & $\begin{array}{l}\text { Tumor: } 1-5 \% \\
\text { immune: } 1-5 \%\end{array}$ & PMS2 loss, confirmed $P M S 2$ mutation \\
\hline 4 & 79 & IB & Grade 2 endometrioid & $\begin{array}{l}\text { Tumor: }>50 \% \\
\text { immune: } 1-5 \%\end{array}$ & $\begin{array}{l}\text { Tumor: }<1 \% \\
\text { immune: } 26-50 \%\end{array}$ & MLH1/PMS2 loss, $M L H 1$ methylation \\
\hline
\end{tabular}

carcinomas. Tumor expression of the immune modulatory enzyme IDO could, for instance, reduce the effectiveness of these therapies by rendering tumor-associated cytotoxic $\mathrm{T}$ cells dysfunctional, as exposing a tumor to immune recognition is of little utility if the immune system cannot effectively eliminate it $[14,15]$.

In its normal function, IDO plays a critical role in maintaining immune balance. It was initially discovered in the placenta, where its expression in vascular and trophoblastic cells promotes fetal tolerance by downregulating local maternal T-cell responses [21-25]. Mouse models suggest that IDO is a key contributor to the immunoregulatory properties of splenic dendritic cells and that its expression on these cells can block clonal T-cell expansion [26]. IDO also seems to play a role in curbing autoimmunity, with IDO inhibition contributing to collageninduced arthritis and autoimmune encephalomyelitis, colitis, and diabetes in experimental models [27-30]. In the transplant setting, IDO appears crucial for allowing graft tolerance, with IDO induction promoting allograft tolerance and curbing graft vs. host lethality [31-33].

IDO is of particular interest in immunotherapeutic cancer treatment because it is one of the only immune targets with clinically available antagonists [15]. Furthermore, IDO has been shown to have relevance in endometrial cancers where its expression correlates with impaired T-cell infiltration and worsened survival [16-18]. Work from Liu et al. [14] revealed IDO expression of at least $1 \%$ in $57 \%$ of endometrial carcinomas, with significantly higher rates $(63 \%$ vs. $38 \%, p<0.01)$ in recurrent vs. primary cancers. Ino et al. identified IDO expression in $49 \%$ of endometrial cancers using a cutoff of 5\% tumoral expression, and found IDO was positively correlated with myometrial invasion, nodal metastasis, lymphovascular space involvement, and elevated $\mathrm{CD}^{+}$and $\mathrm{CD}^{+}$tumor-infiltrating and peritumoral lymphocytes [16, 17]. Furthermore, IDO expression served as an independent prognostic factor for impaired progression-free survival. de Jong et al. [18] identified IDO expression of at least $5 \%$ in a smaller proportion of cases (18.1\%) when compared to these studies, but found a similar association with poor survival.
In our series, IDO was commonly expressed by endometrial carcinomas, with $85 \%$ showing at least focal $(>1 \%)$ tumor staining and $60 \%$ showing at least 5\% staining. Overall, tumoral IDO expression was significantly more common than PD-L1 expression within tumor cells, with almost all PD-L1 positive tumors co-expressing IDO but over half of IDO-positive tumors lacking PD-L1. As has been demonstrated with PD-L1, tumoral IDO expression tended to concentrate at the infiltrating edge of endometrial carcinomas suggesting an ongoing adaptive immune response. The observed rates of IDO expression seen in this series are comparable to what was observed by Liu et al. and Ino et al., but are considerably higher than were noted by de Jong et al. [14, 16-18]. This may be largely attributable to the fact that our study, like the works by Liu and Ino, was performed on whole-tissue sections, whereas the de Jong study was performed on tissue microarray.

To our knowledge, this study represents the first investigation of IDO expression in specific mismatch repair subsets of endometrial carcinoma. Mismatch repair status has been previously linked to PD-L1 expression in colorectal and endometrial carcinomas, and was sufficiently correlated with response to anti-PD-1 therapy to warrant FDA-approval in this setting [1-4, 34]. The increased incidence of immunomodulatory molecule expression in mismatch repair-deficient cancers is thought to be due to the increased neoantigen loads of these tumors, leading to higher levels of immune recognition/infiltration and subsequent development of adaptive mechanisms of tumoral immune evasion [1-4, 34]. While this association has been well-documented for PD-L1, there has been little work done on the relationship between mismatch repair status and IDO. An investigation of medullary colorectal carcinomas by Friedman et al. [34] identified significantly higher IDO expression among microsatellite unstable vs. stable tumors (32\% vs. $3 \%, p<0.0001)$, paralleling what has been observed with PD-L1. Our study yielded similar results: as was seen with PD-L1, IDO was more commonly expressed at high levels in mismatch repair-deficient endometrial cancers when compared to mismatch repair-intact tumors, with expression at the $25 \%$ threshold showing statistical 
significance. Furthermore, Lynch syndrome-associated cancers were significantly more likely than MLH1hypermethylated cases to be $>25 \%$ IDO-positive. Of the only four cases showing $>50 \%$ tumor cell staining for IDO, all were mismatch repair-deficient and three were Lynch syndrome-associated. Although there was no observed association between IDO expression and prognosis in this series, the study is limited by a relatively low proportion of patients $(8 \%)$ with recurrence.

These findings have several implications. First, the high frequency of dual IDO/PD-L1 expression in endometrial cancers suggests that response to therapies targeting the PD-1/PD-L1 axis alone may by muted in this tumor type due to IDO interference with immune cell function. Combined therapies may therefore be of clinical utility in this scenario, as has been proposed in other organs. While there are no current trials available in endometrial cancers specifically, there are several trials of epacadostat, an oral IDO1 inhibitor, in combination with immune checkpoint inhibitors, cancer vaccines, and targeted therapies actively enrolling in a number of disease sites including melanoma, non-small cell lung cancer, colorectal cancer, gastric cancer, pancreatic cancer, urothelial cancer, and ovarian cancer (Clinical trials.gov) [12]. In terms of gynecologic cancers, endometrial cancers have thus far shown the most promise for the success of immunotherapy, given the role for checkpoint inhibition in microsatellite unstable cancers, and should be considered for evaluation of combination therapies.

Second, these data suggest that there may be differences in immunotherapeutic vulnerability within the mismatch repair-deficient umbrella, with Lynch syndrome-associated cancers showing relatively higher expression of antiimmune defense molecules relative to MLH1hypermethylated cancers. This indicates that mismatch repair status alone may not be fully predictive of immunotherapeutic response; rather, that specific molecular alterations may be of interest. It is notable that the most strongly IDO-positive tumors included two PMS2-deficient tumors, one MSH2/MSH6-deficient tumor, and one MLHhypermethylated tumor, whereas prior investigations from our institution revealed that PD-L1 was most strikingly positive in tumors with MSH6 mutations (Sloan et al. [4]). Furthermore, there was no statistically significant correlation between grade and IDO expression, and indeed the most strongly IDO-positive tumors in this series were not high grade or otherwise morphologically remarkable, indicating that morphology is not necessarily the best indication of immunogenicity.

Finally, although IDO shows higher expression overall in mismatch repair-deficient cancers, it remains common in mismatch repair-intact tumors. Targeting IDO may therefore be a viable treatment approach in tumors showing no abnormalities in the mismatch repair system: a group for which immunotherapy is not currently considered a viable option. Further studies investigating treatments targeting IDO in both mismatch repair-deficient and mismatch repairintact endometrial cancers are therefore warranted.

Acknowledgements We thank the University of Virginia Biorepository and Tissue Research Facility for their skill and expertise in performing all immunohistochemical stains.

\section{Compliance with ethical standards}

Conflict of interest The authors declare that they have no conflict of interest.

\section{References}

1. Le DT, Uram JN, Wang H, et al. PD-1 blockade in tumors with mismatch-repair deficiency. N Engl J Med. 2015;372:2509-20.

2. Le DT, Durham JN, Smith KN, et al. Mismatch-repair deficiency predicts response of solid tumors to PD-1 blockade. Science. 2017;357:409-13.

3. Howitt BE, Shukla SA, Sholl LM, et al. Association of polymerase e-mutated and microsatellite-instable endometrial cancers with neoantigen load, number of tumor-infiltrating lymphocytes, and expression of PD-1 and PD-L1. JAMA Oncol. 2015;1:1319-23.

4. Sloan EA, Ring KL, Willis BC, Modesitt SC, Mills AM. PD-L1 expression in mismatch repair-deficient endometrial carcinomas, including Lynch syndrome-associated and MLH1 promoter hypermethylated tumors. Am J Surg Pathol. 2017;41:326-33.

5. Taube JM, Klein A, Brahmer JR, et al. Association of PD-1, PD-1 ligands, and other features of the tumor immune microenvironment with response to anti-PD-1 therapy. Clin Cancer Res. 2014;20:5064-74.

6. Butte MJ, Keir ME, Phamduy TB, Sharpe AH, Freeman GJ. Programmed death-1 ligand 1 interacts specifically with the B7-1 costimulatory molecule to inhibit $\mathrm{T}$ cell responses. Immunity. 2007;27:111-22.

7. Longoria TC, Eskander RN. Immunotherapy in endometrial cancer-an evolving therapeutic paradigm. Gynecol Oncol Res Pract. 2015;2:11.

8. Lee V, Le DT. Efficacy of PD-1 blockade in tumors with MMR deficiency. Immunotherapy. 2016;8:1-3.

9. Reiss KA, Forde PM, Brahmer JR. Harnessing the power of the immune system via blockade of PD-1 and PD-L1: a promising new anticancer strategy. Immunotherapy. 2014;6:459-75.

10. Tumeh PC, Harview CL, Yearley JH, et al. PD-1 blockade induces responses by inhibiting adaptive immune resistance. Nature. 2014;515:568-71.

11. Vanderstraeten A, Luyten C, Verbist G, Tuyaerts S, Amant F. Mapping the immunosuppressive environment in uterine tumors: implications for immunotherapy. Cancer Immunol Immunother. 2014;63:545-57.

12. Hughes PE, Caenepeel S, Wu LC. Targeted therapy and checkpoint immunotherapy combinations for the treatment of cancer. Trends Immunol. 2016;37:462-76.

13. Hanahan D, Weinberg RA. Hallmarks of cancer: the next generation. Cell. 2011;144:646-74.

14. Liu J, Liu Y, Wang W, Wang C, Che Y. Expression of immune checkpoint molecules in endometrial carcinoma. Exp Ther Med. 2015;10:1947-52. 
15. Moon YW, Hajjar J, Hwu P, Naing A. Targeting the indoleamine 2,3-dioxygenase pathway in cancer. $\mathbf{J}$ Immunother Cancer. 2015;3:51.

16. Ino K, Yamamoto E, Shibata K, et al. Inverse correlation between tumoral indoleamine 2,3-dioxygenase expression and tumorinfiltrating lymphocytes in endometrial cancer: its association with disease progression and survival. Clin Cancer Res. 2008;14:2310-7.

17. Ino $\mathrm{K}$, Yoshida $\mathrm{N}$, Kajiyama $\mathrm{H}$, et al. Indoleamine 2,3-dioxygenase is a novel prognostic indicator for endometrial cancer. $\mathrm{Br} \mathbf{~ J}$ Cancer. 2006;95:1555-61.

18. de Jong RA, Kema IP, Boerma A, et al. Prognostic role of indoleamine 2,3-dioxygenase in endometrial carcinoma. Gynecol Oncol. 2012;126:474-80.

19. Topalian SL, Hodi FS, Brahmer JR, et al. Safety, activity, and immune correlates of anti-PD-1 antibody in cancer. N Engl J Med. 2012;366:2443-54.

20. Herbst RS, Soria JC, Kowanetz M, et al. Predictive correlates of response to the anti-PD-L1 antibody MPDL3280A in cancer patients. Nature. 2014;515:563-7.

21. Honig A, Rieger L, Kapp M, Sutterlin M, Dietl J, Kammerer U. Indoleamine 2,3-dioxygenase (IDO) expression in invasive extravillous trophoblast supports role of the enzyme for maternofetal tolerance. J Reprod Immunol. 2004;61:79-86.

22. Munn DH, Mellor AL. IDO in the tumor microenvironment: inflammation, counter-regulation, and tolerance. Trends Immunol. 2016;37:193-207.

23. Munn DH, Mellor AL. Indoleamine 2,3 dioxygenase and metabolic control of immune responses. Trends Immunol. 2013;34:137-43.

24. Munn DH, Zhou M, Attwood JT, et al. Prevention of allogeneic fetal rejection by tryptophan catabolism. Science. 1998;281:1191-3.

25. Suzuki S, Tone S, Takikawa O, Kubo T, Kohno I, Minatogawa Y. Expression of indoleamine 2,3-dioxygenase and tryptophan 2,3dioxygenase in early concepti. Biochem J. 2001;355:425-9.
26. Mellor AL, Baban B, Chandler $\mathrm{P}$, et al. Cutting edge: induced indoleamine 2,3 dioxygenase expression in dendritic cell subsets suppresses $\mathrm{T}$ cell clonal expansion. J Immunol. 2003;171:1652-5.

27. Szanto S, Koreny T, Mikecz K, Glant TT, Szekanecz Z, Varga J. Inhibition of indoleamine 2,3-dioxygenase-mediated tryptophan catabolism accelerates collagen-induced arthritis in mice. Arthritis Res Ther. 2007;9:R50.

28. Gurtner GJ, Newberry RD, Schloemann SR, McDonald KG, Stenson WF. Inhibition of indoleamine 2,3-dioxygenase augments trinitrobenzene sulfonic acid colitis in mice. Gastroenterology. 2003; 125:1762-73.

29. Fallarino F, Volpi C, Zelante T, et al. IDO mediates TLR9-driven protection from experimental autoimmune diabetes. J Immunol. 2009;183:6303-12.

30. Yan Y, Zhang GX, Gran B, et al. IDO upregulates regulatory $\mathrm{T}$ cells via tryptophan catabolite and suppresses encephalitogenic $\mathrm{T}$ cell responses in experimental autoimmune encephalomyelitis. J Immunol. 2010;185:5953-61.

31. Lu Y, Giver CR, Sharma A, et al. IFN-gamma and indoleamine 2,3-dioxygenase signaling between donor dendritic cells and $\mathrm{T}$ cells regulates graft versus host and graft versus leukemia activity. Blood. 2012;119:1075-85.

32. Lan Z, Ge W, Arp J, et al. Induction of kidney allograft tolerance by soluble CD83 associated with prevalence of tolerogenic dendritic cells and indoleamine 2,3-dioxygenase. Transplantation. 2010;90:1286-93.

33. Jasperson LK, Bucher C, Panoskaltsis-Mortari A, et al. Indoleamine 2,3-dioxygenase is a critical regulator of acute graft-versushost disease lethality. Blood. 2008;111:3257-65.

34. Friedman K, Brodsky AS, Lu S, et al. Medullary carcinoma of the colon: a distinct morphology reveals a distinctive immunoregulatory microenvironment. Mod Pathol. 2016;29:528-41. 